\title{
EMBEDDING UNIVALENT FUNCTIONS IN FILTERING LOEWNER CHAINS IN HIGHER DIMENSION
}

\author{
LEANDRO AROSIO, FILIPPO BRACCI, AND ERLEND FORNÆSS WOLD \\ (Communicated by Franc Forstneric)
}

\begin{abstract}
We discuss the problem of embedding univalent functions into Loewner chains in higher dimension. In particular, we prove that a normalized univalent map of the ball in $\mathbb{C}^{n}$ whose image is a smooth strongly pseudoconvex domain is embeddable into a normalized Loewner chain (also satisfying some extra regularity properties) if and only if the closure of the image is polynomially convex.
\end{abstract}

\section{INTRODUCTION}

Let $\mathbb{B}^{n}:=\left\{z \in \mathbb{C}^{n}:\|z\|^{2}<1\right\}$ denote the unit ball of $\mathbb{C}^{n}$. Let

$$
\mathcal{S}:=\left\{f: \mathbb{B}^{n} \rightarrow \mathbb{C}^{n}: f(0)=0, d f_{0}=\mathrm{id}, f \text { univalent }\right\} .
$$

Recall that a normalized Loewner chain $\left(f_{t}\right)$ is a family of univalent mappings $\left(f_{t}: \mathbb{B}^{n} \rightarrow \mathbb{C}^{n}\right)_{t \geq 0}$, with $\Omega_{s}:=f_{s}\left(\mathbb{B}^{n}\right) \subset f_{t}\left(\mathbb{B}^{n}\right)$ for $s \leq t$ and such that $f_{t}(0)=0$ and $d f_{0}=e^{t}$ id for all $t \geq 0$. We set $R\left(f_{t}\right):=\bigcup_{s>0} \Omega_{s} \subseteq \mathbb{C}^{n}$ and call the Loewner range of $\left(f_{t}\right)$ the class of biholomorphism of $R\left(f_{t}\right)$. A normalized Loewner chain $\left(f_{t}\right)$ always has the Loewner range biholomorphic to $\mathbb{C}^{n}$, but in dimension greater than 1, due to the existence of Fatou-Bieberbach domains, the open set $R\left(f_{t}\right)$ might be strictly contained in $\mathbb{C}^{n}$ (see Section 2 for more details about Loewner chains).

We say that a function $f \in \mathcal{S}$ embeds into a normalized Loewner chain if there exists a normalized Loewner chain $\left(f_{t}\right)$ such that $f_{0}=f$. We denote this by

$\mathcal{S}^{1}:=\left\{f \in \mathcal{S}: f\right.$ embeds into a normalized Loewner chain $\left(f_{t}\right)$ with $\left.R\left(f_{t}\right)=\mathbb{C}^{n}\right\}$.

For $n=1$, the class $\mathcal{S}$ is quite well understood by means of the Loewner theory (see, e.g. [18, 14] or the recent survey [1]). In fact, every element $f$ of $\mathcal{S}$ for $n=1$ can be embedded into a normalized Loewner chain whose range is $\mathbb{C}$ (see [18, Thm. 6.1], which by itself can be described by a differential equation with a particular driving term. Thus $\mathcal{S}=\mathcal{S}^{1}$ and such a class is also compact (see 18]).

In higher dimension, in 15, 12, I. Graham, H. Hamada and G. Kohr introduced and studied the class $\mathcal{S}^{0}$ which is the subclass of $\mathcal{S}^{1}$ formed by those $f \in \mathcal{S}$ which admit a parametric representation, namely, such that $f$ can be embedded into a normalized Loewner chain $\left(f_{t}\right)$ with the property that $\left\{e^{-t} f_{t}(\cdot)\right\}_{t \geq 0}$ is a normal

Received by the editors June 26, 2013 and, in revised form, August 21, 2013.

2010 Mathematics Subject Classification. Primary 32H02, 32T15, 32A30, 30C55.

Key words and phrases. Loewner theory, embedding problems, univalent functions, polynomially convex domains, Runge domains.

The first and second authors were supported by ERC grant "HEVO - Holomorphic Evolution Equations" n. 277691.

The third author was supported by NFR grant 209751/F20.

(C)2014 American Mathematical Society Reverts to public domain 28 years from publication 
family. A geometric characterization of maps in the class $\mathcal{S}^{0}$ is in 13. The class $\mathcal{S}^{0}$ is compact and in dimension one it holds $\mathcal{S}^{0}=\mathcal{S}^{1}=\mathcal{S}$, while in higher dimension one has $\mathcal{S}^{0} \subsetneq \mathcal{S}^{1}$ (see [14, Section 8]).

It is then natural to ask whether $\mathcal{S}=\mathcal{S}^{1}$ for $n>1$. It turns out that this is not always the case: since every normalized Loewner chain is a $L^{\infty}$ Loewner chain in the sense of [5] (see Proposition 2.1), as explained in [6, Section 4] it follows from a theorem of Docquier-Grauert [10] that if $f \in \mathcal{S}^{1}$, then $f\left(\mathbb{B}^{n}\right)$ is a Runge domain. Therefore, if $f \in \mathcal{S}$ is such that $f\left(\mathbb{B}^{n}\right)$ is not Runge, then $f \notin \mathcal{S}^{1}$ (and normalized univalent maps of the ball with non-Runge image do exist; see Example 2.2). There is, however, the possibility that $f$ embeds into a Loewner chain whose range is a non-Runge Fatou-Bieberbach domain in $\mathbb{C}^{n}$. Thus the two following natural questions arise:

Q1) Does any $f \in \mathcal{S}$ with $f\left(\mathbb{B}^{n}\right)$ Runge embed into a normalized Loewner chain $\left(f_{t}\right)$ with $R\left(f_{t}\right)=\mathbb{C}^{n}$ ?

Q2) Does any $f \in \mathcal{S}$ embed into a normalized Loewner chain whose Loewner range is biholomorphic to $\mathbb{C}^{n}$ ?

In this note we discuss the two questions and give a partial positive answer to question Q1. Let us denote it by $\mathcal{S}_{R}:=\left\{f \in \mathcal{S}: f\left(\mathbb{B}^{n}\right)\right.$ is Runge $\}$. Question Q1 can then be restated in the following way: is $\mathcal{S}^{1}=\mathcal{S}_{R}$ ? Using the Andersén-Lempert approximation theorem [2, Thm. 2.1] it is proved in [17, Theorem 2.3] that

$$
\overline{\mathcal{S}^{1}}=\mathcal{S}_{R},
$$

where the closure has to be understood in terms of the topology of uniform convergence on compacta of $\mathbb{B}^{n}$. However, one can say more: each $f \in \mathcal{S}_{R}$ can be approximated by maps in $\mathcal{S}$ which can be embedded into "nice" normalized Loewner chains whose range is $\mathbb{C}^{n}$.

Definition 1.1. Let $\left(f_{t}\right)$ be a normalized Loewner chain in $\mathbb{B}^{n}$. We say that $\left(f_{t}\right)$ is a filtering normalized Loewner chain provided the family $\left(\Omega_{t}\right)_{t>s}$ is a neighborhood basis for $\bar{\Omega}_{s}$ for all $s \geq 0$, i.e., the following conditions hold:

(M1) $\bar{\Omega}_{s} \subset \Omega_{t}$ for all $t>s$ and

(M2) for any open set $U$ containing $\bar{\Omega}_{s}$ there exists $t_{0}>s$ such that $\Omega_{t} \subset U$ for all $t \in\left(s, t_{0}\right)$.

As a matter of notation, we let

$\mathcal{S}_{\mathfrak{F}}^{1}:=\left\{f \in \mathcal{S}: f\right.$ embeds in a filtering normalized Loewner chain with $\left.R\left(f_{t}\right)=\mathbb{C}^{n}\right\}$.

Clearly, $\mathcal{S}_{\mathfrak{F}}^{1} \subset \mathcal{S}^{1} \subset \mathcal{S}_{R}$ and $\overline{\mathcal{S}_{\mathfrak{F}}^{1}}=\mathcal{S}_{R}$ (see Corollary 3.3). The main result of this note is the following:

Theorem 1.2. Let $n \geq 2$ and let $f \in \mathcal{S}_{R}$. Assume that $\Omega:=f\left(\mathbb{B}^{n}\right)$ is a bounded strongly pseudoconvex domain with $\mathcal{C}^{\infty}$ boundary. Then $f \in \mathcal{S}_{\mathfrak{F}}^{1}$ if and only if $\bar{\Omega}$ is polynomially convex.

The proof of Theorem 1.2 is the content of Section 3 Finally, in Section 4 we make some final remarks and discuss the role of polynomial convexity in the embedding problem, constructing various examples which explain the role of the hypotheses. 


\section{LOEWNER CHAins AND LOEWNER RANGE}

A general Loewner theory in the unit disc and hyperbolic manifolds was introduced in [5, 7, 9] (see also [3, 4]). According to [5, a Loewner chain of order $d \in[1, \infty]$ in $\mathbb{C}^{n}$ is a family of univalent mappings $\left(f_{t}: \mathbb{B}^{n} \rightarrow \mathbb{C}^{n}\right)_{t \in[0, \infty)}$, with $\Omega_{s}:=f_{s}\left(\mathbb{B}^{n}\right) \subset f_{t}\left(\mathbb{B}^{n}\right)$ for $s \leq t$, such that for any compact set $K \subset \mathbb{B}^{n}$ it holds that

$$
\left|f_{s}(z)-f_{t}(z)\right| \leq \int_{s}^{t} k_{K}(\zeta) d \zeta, \text { for some } k_{K} \in L_{\text {loc }}^{d}\left([0,+\infty), \mathbb{R}^{+}\right), \forall z \in K
$$

The set $R\left(f_{t}\right):=\bigcup_{s \geq 0} \Omega_{s}$ is a domain in $\mathbb{C}^{n}$ whose class of biholomorphism is called the Loewner range of $\left(f_{t}\right)$. The Loewner range of a Loewner chain is an invariant for the associated Loewner equation (see [5], 6]).

The normalization required in the definition of a normalized Loewner chain forces such a family to be a Loewner chain of order $\infty$ with range biholomorphic to $\mathbb{C}^{n}$ :

Proposition 2.1. Let $\left(f_{t}\right)$ be a normalized Loewner chain. Then $\left(f_{t}\right)$ is a Loewner chain of order $\infty$ and its Loewner range is biholomorphic to $\mathbb{C}^{n}$.

Moreover, if the family $\left\{e^{-t} \circ f_{t}\right\}_{t \geq 0}$ is uniformly bounded in a neighborhood of the origin, then $R\left(f_{t}\right)=\mathbb{C}^{n}$.

Proof. According to [14, Theorem 8.1.8], the family $\left(f_{t}\right)$ is locally Lipschitz continuous in $t$ locally uniformly with respect to $z$, hence it satisfies (2.1) with $k_{K}$ a positive constant. Therefore $\left(f_{t}\right)$ is a Loewner chain of order $\infty$.

The family $\left(\varphi_{s, t}: \mathbb{B}^{n} \rightarrow \mathbb{B}^{n}\right)_{0 \leq s \leq t}$ defined as $\varphi_{s, t}:=f_{t}^{-1} \circ f_{s}$ for all $0 \leq s \leq t$ is an evolution family (of order $\infty$ according to [5]) which satisfies

$$
\varphi_{s, t}(z)=e^{s-t} z+O\left(|z|^{2}\right), \quad 0 \leq s \leq t .
$$

By [14, Theorem 8.1.5] there exists a normalized Loewner chain $\left(g_{t}: \mathbb{B}^{n} \rightarrow \mathbb{C}^{n}\right)$ associated with $\left(\varphi_{s, t}\right)$ such that $R\left(g_{t}\right)=\mathbb{C}^{n}$. But then $R\left(f_{t}\right)$ is biholomorphic to $\mathbb{C}^{n}$ by [5, Corollary 4.8].

Let $h_{t}:=e^{-t} \circ f_{t}, t \geq 0$. If $\left\{h_{t}\right\}_{t \geq 0}$ is uniformly bounded in a neighborhood of the origin, then there exists a ball $s \mathbb{B} \subset \bigcap_{t \in \mathbb{R}^{+}} h_{t}\left(\mathbb{B}^{n}\right)$, and thus

$$
\mathbb{C}^{n}=\bigcup_{t \in \mathbb{R}^{+}} e^{t}(s \mathbb{B}) \subset \bigcup_{t \in \mathbb{R}^{+}} e^{t}\left(h_{t}\left(\mathbb{B}^{n}\right)\right),
$$

as claimed.

In [6. Section 4] it has been shown that if $\left(f_{t}\right)$ is a Loewner chain of order $d$, then $\left(\Omega_{s}, \Omega_{t}\right)$ is a Runge pair for all $0 \leq s \leq t$ and, as a consequence, $\Omega_{t}$ is Runge in $R\left(f_{t}\right)$ for all $t \geq 0$. If $\left(f_{t}\right)$ is a normalized Loewner chain, then $R\left(f_{t}\right)$ is biholomorphic to $\mathbb{C}^{n}$, but it might happen that $\Omega_{t}$ is not Runge in $\mathbb{C}^{n}$ :

Example 2.2. Let $D$ be a Fatou-Bieberbach domain of $\mathbb{C}^{2}$ which is not Runge (see [19]), assume $0 \in D$ and let $\phi: \mathbb{C}^{n} \rightarrow D$ be a biholomorphism such that $\phi(0)=0$ and $d \phi_{0}=\mathrm{id}$. Then $f_{t}(z):=\phi\left(e^{t} z\right), t \geq 0, z \in \mathbb{B}^{n}$, is a normalized Loewner chain. Note that $R\left(f_{t}\right)=D$ is not Runge in $\mathbb{C}^{n}$, hence $\Omega_{t}$ is not Runge in $\mathbb{C}^{n}$ for $t$ sufficiently big. 


\section{EMbedding INTO FILTERING LOEWNER CHAins}

We first make the following simple observations.

Remark 3.1. If $f \in \mathcal{S}_{\mathfrak{F}}^{1}$, then $\overline{f\left(\mathbb{B}^{n}\right)}$ is polynomially convex, since it has a Stein and Runge neighborhood basis given by $\left(\Omega_{t}\right)_{t>0}$.

A domain $D \subset \mathbb{C}^{n}$ is called convexshapelike if there exists an automorphism $\psi \in A u t_{h o l} \mathbb{C}^{n}$ such that $\psi(D)$ is convex in $\mathbb{C}^{n}$.

Proposition 3.2. Let $f \in \mathcal{S}_{R}$, and let $\Omega:=f\left(\mathbb{B}^{n}\right)$. If $\Omega$ is a bounded convexshapelike domain, then $f \in \mathcal{S}_{\mathfrak{F}}^{1}$.

Proof. Assume $\Omega$ is a convexshapelike domain. Let $\psi \in A u t_{h o l} \mathbb{C}^{n}$ be such that $\psi(0)=0$ and $\psi(\Omega)$ is convex. Hence the family of univalent mappings $f_{t}(z):=$ $\psi^{-1}\left(e^{t} \cdot \psi(f(z))\right)$ has the property $f_{s}\left(\mathbb{B}^{n}\right) \subset f_{t}\left(\mathbb{B}^{n}\right)$ for all $0 \leq s \leq t$, and it is easy to check that it forms a filtering normalized Loewner chain such that $f_{0}=f$.

Corollary 3.3. $\overline{\mathcal{S}_{\mathfrak{F}}^{1}}=\mathcal{S}_{R}$.

Proof (cf. also [17, Thm 2.3]). Let $f \in \mathcal{S}_{R}$. By the Andérsen-Lempert theorem [2. Thm. 2.1] there exists a sequence $\left\{\psi_{m}\right\}_{m \in \mathbb{N}}$ of automorphisms of $\mathbb{C}^{n}$ such that $\psi_{m} \rightarrow f$ uniformly on compacta of $\mathbb{B}^{n}$. We can assume that $\psi_{m}(0)=0$ and $d\left(f_{m}\right)_{0}=$ id for all $m \geq 0$. Since $\psi_{m}\left(\mathbb{B}^{n}\right)$ is by construction a bounded convexshapelike domain, we have that $\left.\psi_{m}\right|_{\mathbb{B}^{n}} \in \mathcal{S}_{\mathfrak{F}}^{1}$.

Remark 3.4. Let $\Omega \Subset \mathbb{C}^{n}$ be a convexshapelike domain. Then $\Omega$ is Runge and $\bar{\Omega}$ is polynomially convex. Indeed, it is clear that $\Omega$ is Runge because convex domains are Runge and Runge-ness is invariant under automorphisms of $\mathbb{C}^{n}$. Moreover, if $\psi \in A u t_{h o l} \mathbb{C}^{n}$ is such that $0 \in \psi(\Omega)$ and $\psi(\Omega)$ is convex, then $\left\{\psi^{-1}(t \psi(\Omega))\right\}_{t>1}$ is a Runge and Stein neighborhood basis of $\bar{\Omega}$, which is thus polynomially convex ([16, Thm. 1.11]).

The proof of Theorem 1.2 depends on the following well known Mergelyan type result, for which we give a proof for lack of a suitable reference.

Lemma 3.5. Let $\Omega \subset \mathbb{C}^{n}$ be a bounded strongly pseudoconvex domain with $\mathcal{C}^{\infty}$ boundary which is biholomorphic to $\mathbb{B}^{n}$. Then any $f \in \mathcal{C}^{2}(\bar{\Omega}) \cap \mathcal{O}(\Omega)$ can be approximated uniformly on $\bar{\Omega}$ in $\mathcal{C}^{2}$-norm, by functions in $\mathcal{O}(\bar{\Omega})$.

Proof. Let $X$ denote the radial vector field $X(z)=-\sum_{j=1}^{n} z_{j} \frac{\partial}{\partial z_{j}}$ defined on $\overline{\mathbb{B}^{n}}$. By Fefferman's theorem 11 the biholomorphism between the ball and $\Omega$ extends smoothly to the boundary, and we can push $X$ forward to $\bar{\Omega}$ in order to get a vector field $\tilde{X}$ pointing into $\Omega$ on $\partial \Omega$. By [16, Thm. 2.1 p. 280], $\tilde{X}$ may be approximated uniformly on $\bar{\Omega}$ by vector fields in $\mathcal{O}(\bar{\Omega})$. In particular, there exists a vector field $Y \in \mathcal{O}(\bar{\Omega})$ pointing into $\Omega$ on $\partial \Omega$. Hence its real flow $\varphi_{t}$ is well defined for all $t \geq 0$ and $\varphi_{t}(\bar{\Omega}) \subset \Omega$ for $t>0$.

Let $f \in \mathcal{C}^{2}(\bar{\Omega}) \cap \mathcal{O}(\Omega)$. Then $f \circ \varphi_{t} \in \mathcal{O}(\bar{\Omega})$ for all $t>0$, and $f \circ \varphi_{t} \rightarrow f$ in $\mathcal{C}^{2}$-norm uniformly on $\bar{\Omega}$ as $t \rightarrow 0^{+}$.

The proof of our result relies also on the following result, which might be interesting on its own. 
Proposition 3.6. Let $n \geq 2$. Let $\Omega \subset \mathbb{C}^{n}$ be a bounded strongly pseudoconvex domain with $\mathcal{C}^{\infty}$ boundary which is biholomorphic to $\mathbb{B}^{n}$. Then the following are equivalent:

(1) $\bar{\Omega}$ is polynomially convex,

(2) $\Omega$ is convexshapelike.

Moreover, if condition (1) or (2) holds, then $\Omega$ is Runge.

Proof. $(2) \Rightarrow(1)$ it follows from Remark 3.4 (and also implies that $\Omega$ is Runge).

$(1) \Rightarrow(2)$. Assume that $\bar{\Omega}$ is polynomially convex. The aim is to find $\psi \in$ $A u t_{h o l} \mathbb{C}^{n}$ such that $\psi(\Omega)$ is a convex domain (in fact a strongly convex domain with smooth boundary).

In order to find such an automorphism $\psi$, we are going to find an open neighborhood $U$ of $\bar{\Omega}$ and an univalent map $h: U \rightarrow \mathbb{C}^{n}$ such that

(1) $U$ is Runge,

(2) $h(U)$ is starlike (in fact, convex),

(3) $h(\Omega)$ is a strongly convex domain with smooth boundary.

Once we have that, by the Andersén-Lempert theorem [2, Thm. 2.1] we can approximate $h^{-1}$ on $h(U)$-and hence $h$ on $U$ - uniformly on compacta with automorphisms of $\mathbb{C}^{n}$. Hence we can find an automorphism $\psi$ having the required properties, and we are done.

In order to construct $h$, let $f: \mathbb{B}^{n} \rightarrow \Omega$ be a biholomorphism. We note that by Fefferman's theorem 11$] f$ extends to a diffeomorphism $f: \overline{\mathbb{B}^{n}} \rightarrow \bar{\Omega}$. By Lemma 3.5. $f^{-1}$ can be approximated in $\mathcal{C}^{2}$-norm uniformly on $\bar{\Omega}$ by holomorphic maps defined on neighborhoods of $\bar{\Omega}$. Therefore, there exists an open neighborhood $U^{\prime}$ of $\bar{\Omega}$ and a univalent map $h: U^{\prime} \rightarrow \mathbb{C}^{n}$ such that $h(\Omega)$ is a smooth strongly convex domain. Since a compact polynomially convex set admits a basis of Stein neighborhoods that are Runge in $\mathbb{C}^{n}$, and by hypothesis $\bar{\Omega}$ is polynomially convex, we can find an open set $U^{\prime \prime}$ such that $U^{\prime \prime}$ is Runge and $\bar{\Omega} \subset U^{\prime \prime} \subset U^{\prime}$. Now, since $h(\bar{\Omega})$ has a basis of convex neighborhoods, we can find a convex set $A$ such that $h(\bar{\Omega}) \subset A \subset h\left(U^{\prime \prime}\right)$. Therefore, $U:=h^{-1}(A)$ is Runge in $U^{\prime \prime}$, and since the latter is Runge in $\mathbb{C}^{n}$, it follows that $U$ is Runge in $\mathbb{C}^{n}$, and the proof is concluded.

Remark 3.7. Notice that Proposition 3.6 is false in dimension one, because the group of automorphisms of $\mathbb{C}$ is too "small".

The proof of Theorem 1.2 is now straightforward:

Proof of Theorem 1.2 . If $\bar{\Omega}$ is polynomially convex, then by Proposition 3.6 it follows that $\Omega$ is convexshapelike and hence by Proposition 3.2 it follows that $f \in \mathcal{S}_{\mathfrak{F}}^{1}$. The converse is Remark 3.1 .

Remark 3.8. As an application, we can give an alternative proof of Corollary 3.3 , more in the spirit of the one-dimensional proof by Ch. Pommerenke ([18, Thm. $6.1])$. Thus let $f \in \mathcal{S}_{R}$, and consider $f_{r}(z):=\frac{1}{r} f(r z)$, for $0<r<1$. Then clearly $f_{r} \in \mathcal{S}, f_{r}$ converges uniformly on compacta of $\mathbb{B}^{n}$ to $f$, and $f_{r}\left(\mathbb{B}^{n}\right)$ is a strongly pseudoconvex domain with smooth boundary. Since $\frac{1}{r} f\left(\ell \mathbb{B}^{n}\right)$ with $r<\ell \leq 1$ is a basis of Stein neighborhoods of $\overline{f_{r}\left(\mathbb{B}^{n}\right)}$ that are Runge in $\mathbb{C}^{n}$ (because $f\left(\ell \mathbb{B}^{n}\right)$ is Runge in $f\left(\mathbb{B}^{n}\right)$, which by hypothesis is Runge in $\left.\mathbb{C}^{n}\right)$, it follows that $\overline{f_{r}\left(\mathbb{B}^{n}\right)}$ is polynomially convex. Hence $f_{r} \in \mathcal{S}_{\mathfrak{F}}^{1}$. 


\section{REMARKS AND EXAMPLES}

Let $f \in \mathcal{S}_{R}$. By Remark [3.1, a necessary condition for having $f \in \mathcal{S}_{\mathfrak{F}}^{1}$ is that $\overline{f\left(\mathbb{B}^{n}\right)}$ is polynomially convex. Such a condition, as in the one-dimensional case, is not necessary for having $f \in \mathcal{S}^{1}$, as the following example shows.

Example 4.1. Let $\phi: \mathbb{D} \rightarrow \mathbb{C}$ be a univalent map, $\phi(0)=0, \phi^{\prime}(0)=1$. Then $f(z, w)=(\phi(z), w) \in \mathcal{S}$. Let $\left(\phi_{t}\right)_{t \geq 0}$ be a normalized Loewner chain in $\mathbb{D}$ such that $\phi_{0}=\phi$. Define $f_{t}(z, w):=\left(\phi_{t}(z), e^{t} w\right)$ for $(z, w) \in \mathbb{B}^{2}$. Then $\left(f_{t}\right)$ is a normalized Loewner chain such that $f_{0}=f$. Indeed, setting $\varphi_{s, t}(z, w):=\left(\phi_{t}^{-1} \circ \phi_{s}(z), e^{s-t} w\right)$, by the Schwarz lemma

$$
\left|\phi_{t}^{-1} \circ \phi_{s}(z)\right|^{2}+\left|e^{s-t} w\right|^{2} \leq|z|^{2}+|w|^{2}<1,
$$

from which it follows that $\varphi_{s, t}\left(\mathbb{B}^{2}\right) \subset \mathbb{B}^{2}$, and $f_{t} \circ \varphi_{s, t}=f_{s}$ for $0 \leq s<t$, which implies that $f_{s}\left(\mathbb{B}^{2}\right) \subset f_{t}\left(\mathbb{B}^{2}\right)$ for $0 \leq s<t$.

Now, recall that a compact set $K \subset \mathbb{C}$ is polynomially convex if and only if $\mathbb{C} \backslash K$ is simply connected. Let $D \subset \mathbb{C}$ be a simply connected domain such that $\mathbb{C} \backslash \bar{D}$ is not simply connected (for instance take $D=\{\zeta \in \mathbb{D}:|\zeta-1 / 2|>1 / 2\}$ ). Let $\phi: \mathbb{D} \rightarrow D$ be a Riemann mapping. Up to rescaling, we can assume $\phi(0)=0$ and $\phi^{\prime}(0)=1$. Let $f: \mathbb{B}^{2} \rightarrow \mathbb{B}^{2}$ be defined by $f(z, w):=(\phi(z), w)$. Then $f \in \mathcal{S}^{1}$ but $\overline{f\left(\mathbb{B}^{2}\right)}$ is not polynomially convex.

Next we construct an example of a map $f \in \mathcal{S}_{R}$ such that $f$ extends holomorphically through $\partial \mathbb{B}^{2}$ but $\overline{f\left(\mathbb{B}^{2}\right)}$ is not polynomially convex, and hence $f \notin \mathcal{S}_{\mathfrak{F}}^{1}$.

Example 4.2. In order to construct such an $f$, let $\varphi: \mathbb{B}^{2} \rightarrow \mathbb{C}^{2}$ be a univalent mapping such that $\varphi\left(\mathbb{B}^{2}\right)$ is not a Runge domain (the existence of $\varphi$ follows from the existence of a non-Runge Fatou-Bieberbach domain in $\left.\mathbb{C}^{2}[19]\right)$. Assume $\varphi(0)=0$ and $d \varphi_{0}=$ id.

Let

$$
\vartheta:=\sup \left\{t: \overline{\varphi\left(r \mathbb{B}^{2}\right)} \text { is polynomially convex for all } r<t\right\} .
$$

Then, since $\overline{\varphi\left(r \mathbb{B}^{2}\right)}$ is polynomially convex for $r$ small enough, one has $\vartheta>0$. Moreover, $\vartheta<1$ since $\varphi\left(\mathbb{B}^{2}\right)$ is not Runge (and thus it cannot admit any growing exhaustion by polynomially convex sets). We claim that $K:=\overline{\varphi\left(\vartheta \mathbb{B}^{2}\right)}$ is not polynomially convex. Assume it is, by contradiction. Then $K$ admits a Runge neighborhood basis, and thus there exists a Runge domain $\Omega$ such that

$$
K \subset \Omega \subset \varphi\left(\mathbb{B}^{2}\right) .
$$

Let $\vartheta<u<1$ be such that $\overline{\varphi\left(u \mathbb{B}^{2}\right)} \subset \Omega$. One has that $\overline{u \mathbb{B}^{2}}$ is holomorphically convex in $\mathbb{B}^{2}$, and thus $\overline{\varphi\left(u \mathbb{B}^{2}\right)}$ is holomorphically convex in $\Omega$; hence it is polynomially convex, contradicting the assumption that $\vartheta$ was the supremum.

Finally, define $f(z):=\frac{1}{\vartheta} \varphi(\vartheta z)$. Since $\overline{f\left(\mathbb{B}^{2}\right)}$ is not polynomially convex, it follows from Remark 3.1 that $f \notin \mathcal{S}_{\mathfrak{F}}^{1}$.

We do not know whether $f \in \mathcal{S}^{1}$.

There are also examples of maps $f \in \mathcal{S}^{1}$ such that $\overline{f\left(\mathbb{B}^{n}\right)}$ is polynomially convex but $f \notin \mathcal{S}_{\mathfrak{F}}^{1}$ (and $f\left(\mathbb{B}^{n}\right)$ does not have smooth boundary). A simple example in dimension one is given by a Riemann mapping $f$ from the unit disc $\mathbb{D}$ onto a disc $D$ minus a slit. If such a function were embeddable into a filtering Loewner chain $\left(f_{t}\right)$, then for all $t>0$ the closed disc $\bar{D}$ would be contained in $f_{t}(\mathbb{D})$; hence, by the Carathéodory kernel convergence theorem, $\bar{D} \subset f_{0}(\mathbb{D})$, a contradiction. 
We end this note with the following consideration about Question Q2:

Remark 4.3. Let $f \in \mathcal{S}$. Assume that $f\left(\mathbb{B}^{n}\right)$ is not Runge but suppose there exists a Fatou-Bieberbach $D \subset \mathbb{C}^{n}$ such that $0 \in f\left(\mathbb{B}^{n}\right) \subset D$ and $f\left(\mathbb{B}^{n}\right)$ is Runge in $D$. Then let $F: D \rightarrow \mathbb{C}^{n}$ be a biholomorphism such that $F(0)=0, d F_{0}=$ id. It follows that $F \circ f \in \mathcal{S}_{R}$. Thus, if $\left(g_{t}\right)$ is a normalized Loewner chain with range $\mathbb{C}^{n}$ such that $g_{0}=F \circ f$, then $\left(f_{t}:=F^{-1} \circ g_{t}\right)$ is a normalized Loewner chain with $R\left(f_{t}\right)=D$ such that $f_{0}=f$.

\section{ACKNOWLEGEMENT}

We thank the referee for helpful comments which improved the original manuscript.

\section{REFERENCES}

[1] Marco Abate, Filippo Bracci, Manuel D. Contreras, and Santiago Díaz-Madrigal, The evolution of Loewner's differential equations, Eur. Math. Soc. Newsl. 78 (2010), 31-38. MR2768999

[2] Erik Andersén and László Lempert, On the group of holomorphic automorphisms of $\mathbf{C}^{n}$, Invent. Math. 110 (1992), no. 2, 371-388, DOI 10.1007/BF01231337. MR1185588(93i:32038)

[3] Leandro Arosio, Loewner equations on complete hyperbolic domains, J. Math. Anal. Appl. 398 (2013), no. 2, 609-621, DOI 10.1016/j.jmaa.2012.09.018. MR2990086

[4] Leandro Arosio and Filippo Bracci, Infinitesimal generators and the Loewner equation on complete hyperbolic manifolds, Anal. Math. Phys. 1 (2011), no. 4, 337-350, DOI 10.1007/s13324-011-0020-3. MR2887104

[5] Leandro Arosio, Filippo Bracci, Hidetaka Hamada, and Gabriela Kohr, An abstract approach to Loewner chains, J. Anal. Math. 119 (2013), 89-114, DOI 10.1007/s11854-013-0003-4. MR.3043148

[6] Leandro Arosio, Filippo Bracci, and Erlend Fornæss Wold, Solving the Loewner PDE in complete hyperbolic starlike domains of $\mathbb{C}^{N}$, Adv. Math. 242 (2013), 209-216, DOI 10.1016/j.aim.2013.02.024. MR.3055993

[7] Filippo Bracci, Manuel D. Contreras, and Santiago Díaz-Madrigal, Evolution families and the Loewner equation I: the unit disc, J. Reine Angew. Math. 672 (2012), 1-37. MR2995431

[8] Filippo Bracci, Manuel D. Contreras, and Santiago Díaz-Madrigal, Evolution families and the Loewner equation. II. Complex hyperbolic manifolds, Math. Ann. 344 (2009), no. 4, 947-962, DOI 10.1007/s00208-009-0340-x. MR2507634(2010f:32020)

[9] Manuel D. Contreras, Santiago Díaz-Madrigal, and Pavel Gumenyuk, Loewner chains in the unit disk, Rev. Mat. Iberoam. 26 (2010), no. 3, 975-1012, DOI 10.4171/RMI/624. MR2789373(2012a:30052)

[10] Ferdinand Docquier and Hans Grauert, Leisches Problem und Rungescher Satz für Teilgebiete Steinscher Mannigfaltigkeiten (German), Math. Ann. 140 (1960), 94-123. MR0148939 (26 \#6435)

[11] Charles Fefferman, The Bergman kernel and biholomorphic mappings of pseudoconvex domains, Invent. Math. 26 (1974), 1-65. MR0350069 (50 \#2562)

[12] Ian Graham, Hidetaka Hamada, and Gabriela Kohr, Parametric representation of univalent mappings in several complex variables, Canad. J. Math. 54 (2002), no. 2, 324-351, DOI 10.4153/CJM-2002-011-2. MR1892999 (2003b:32018)

[13] Ian Graham, Hidetaka Hamada, Gabriela Kohr, and Mirela Kohr, Parametric representation and asymptotic starlikeness in $\mathbb{C}^{n}$, Proc. Amer. Math. Soc. 136 (2008), no. 11, 3963-3973, DOI 10.1090/S0002-9939-08-09392-1. MR2425737 (2009e:32016)

[14] Ian Graham and Gabriela Kohr, Geometric function theory in one and higher dimensions, Monographs and Textbooks in Pure and Applied Mathematics, vol. 255, Marcel Dekker Inc., New York, 2003. MR2017933 (2004i:32002)

[15] Gabriela Kohr, Using the method of Löwner chains to introduce some subclasses of biholomorphic mappings in $\mathbf{C}^{n}$, Rev. Roumaine Math. Pures Appl. 46 (2001), no. 6, 743-760 (2002). MR1929522 (2003h:32022) 
[16] R. Michael Range, Holomorphic functions and integral representations in several complex variables, Graduate Texts in Mathematics, vol. 108, Springer-Verlag, New York, 1986. MR.847923 (87i:32001)

[17] S. Schleißinger, On support points on the class $S^{0}\left(\mathbb{B}^{n}\right)$, Proc. Amer. Math. Soc., 142 (2014), no. 11, 3881-3887. MR3251727

[18] Christian Pommerenke, Univalent functions, Vandenhoeck \& Ruprecht, Göttingen, 1975. With a chapter on quadratic differentials by Gerd Jensen; Studia Mathematica/Mathematische Lehrbücher, Band XXV. MR0507768 (58 \#22526)

[19] Erlend Fornæss Wold, A Fatou-Bieberbach domain in $\mathbb{C}^{2}$ which is not Runge, Math. Ann. 340 (2008), no. 4, 775-780, DOI 10.1007/s00208-007-0168-1. MR2372737(2009c:32019)

Dipartimento Di Matematica, Università di Roma "Tor Vergata”, Via Della Ricerca SCIENTIFicA 1, 00133, Roma, ItAly

E-mail address: arosio@mat.uniroma2.it

Dipartimento Di Matematica, Università di Roma "Tor Vergata", Via Della Ricerca Scientifica 1, 00133, Roma, Italy

E-mail address: fbracci@mat.uniroma2.it

Matematisk Institutt, Universitetet i Oslo, Postboks 1053 Blindern, 0316 Oslo, NorWAY

E-mail address: erlendfw@math.uio.no 